\title{
Low angular-dispersion microwave absorption of a dual-pitch nondiffracting metal bigrating
}

\author{
Matthew J. Lockyear, ${ }^{\text {a) }}$ Alastair P. Hibbins, and J. Roy Sambles \\ Thin Film Photonics Group, School of Physics, University of Exeter, Exeter, EX4 4QL United Kingdom \\ Christopher R. Lawrence \\ QinetiQ Ltd., Farnborough, GU14 OLX United Kingdom
}

(Received 6 December 2002; accepted 19 May 2003)

\begin{abstract}
The surface plasmon modes supported by a nondiffracting $90^{\circ}$ bigrating consisting of three grooves per repeat period with one slightly shallower than the other two are characterized by studying the reflectivity from the structure as a function of the angle of incidence and the incident wavelength $\left(11.3<\lambda_{0}<16.7 \mathrm{~mm}\right)$. This structure supports two remarkably angle-independent modes plus a further, lower-energy mode which is more dispersive. Experimental reflectivity is compared with that calculated using a finite element model. In addition, to understand the character of each of the modes, the spatial form of the electromagnetic fields at the resonant frequencies are explored.

(C) 2003 American Institute of Physics. [DOI: 10.1063/1.1593229]
\end{abstract}

Since the initial observations of Wood, ${ }^{1}$ the propagation of surface plasmons (SP) at a planar metal-dielectric interface has been extensively studied and is well understood. ${ }^{2}$ In addition, there exists a substantial body of work concerning the use of periodic surface profiles to couple radiation to SP resonances. ${ }^{3}$ A recent examination into the use of multiperiodic grating profiles to give both resonant modes and to allow simultaneous control of coupling strength has generated much interest. Theoretical work ${ }^{4}$ has shown that it is possible to couple to flatbanded SP branches which normally exist beyond the light cone by convolving an originally sinusoidal profile with a shallower oscillation of longer pitch. Introduction of this secondary pitch results in successive $\boldsymbol{k}_{g}$ scatterings that effectively fold a section of the SP dispersion curve back within the light cone thereby permitting radiative coupling. This coupling has been observed ${ }^{5}$ using a dual pitch square wave metallic grating in the microwave regime. Here we present the bigrating as an extension to this work, with the addition of a second identical profile running at $90^{\circ}$ to the original. Such a bigrating has the potential to act as a polarization independent selective absorber. ${ }^{6,7}$

The grating profile of two deep $(2.24 \mathrm{~mm})$ and one slightly shallower $(1.94 \mathrm{~mm})$ evenly spaced rectangular grooves per repeat period (producing to first order a deep grating profile of $\lambda_{g} / 3$ convolved with a shallower grating of period $\lambda_{g}=7.2 \mathrm{~mm}$ ) were milled in orthogonal directions on an aluminum alloy plate of dimensions $10 \times 500 \times 500 \mathrm{~mm}$. The angle of incidence $\theta$ and the azimuthal angle $\phi$ are shown in Fig. 1 with $\phi=0^{\circ}$ when one of the symmetry planes coincides with the plane of incidence (classical mount). Resonant modes may be observed as minima in the angle-dependant reflectivity with the resonance width being dependent upon the loss channels available. The grating under study does not support propagating diffracted orders over the selected frequency range due to the subwavelength periodicity of the grating. Hence to readily observe the resonant

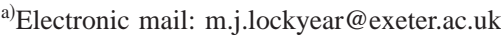

modes in reflectivity the grating grooves are filled with petroleum wax, which is slightly absorbing ${ }^{8}$ at these frequencies. A collimated beam of microwave radiation ${ }^{9}$ $\left(18.0<f_{0}<26.5 \mathrm{GHz}\right.$ with a resolution of $0.02 \mathrm{GHz}$ ) is directed onto the sample, which is mounted upon a rotating turntable, providing data for $0^{\circ} \leqslant \phi \leqslant 360^{\circ}$ at fixed values of $\theta$. The signal is normalized to the reflectivity of a flat plate of the same material and surface plane dimensions as the substrate. Both the source antenna and detector may be set to pass either $p$-polarized (transverse magnetic) or $s$-polarized (transverse electric) radiation allowing reflectivity measurements of $R_{\mathrm{pp}}, R_{\mathrm{ps}}, R_{\mathrm{sp}}$, and $R_{\mathrm{ss}}$ (the subscripts refer to the source and detector polarizations, respectively).

Figure 2 shows the $p$-polarized frequency-dependent reflectivity data obtained for $\theta=52^{\circ}$. The modeled response of the structure is obtained using a finite element code. ${ }^{10} \mathrm{Good}$ agreement is obtained between the theoretical model and the data even though the model assumes a perfect plane wave (the experimental incident angle spread is approximately

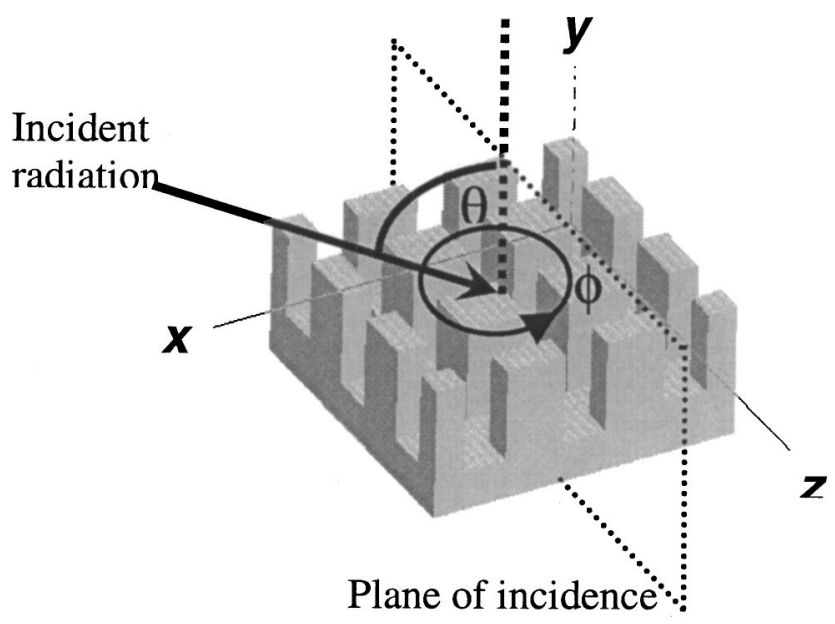

FIG. 1. The unit cell and coordinate system illustrating the polar angle $\theta$, azimuth angle $\phi$, and the plane of incidence. 


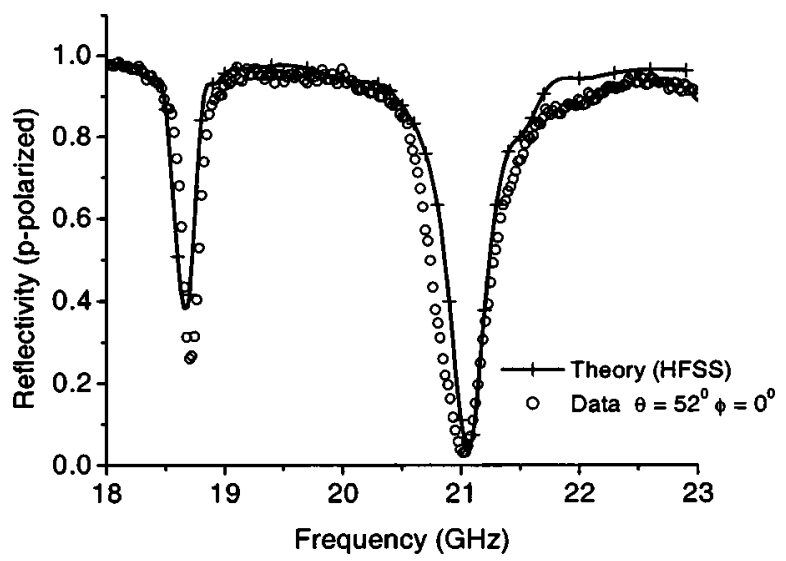

FIG. 2. $p$-polarized experimental and modelled frequency-dependent reflectivity data obtained for $\theta=52^{\circ}$.

$\left.1^{\circ}\right)^{9}$ and treats the sample as infinite in the surface plane. The grating in this instance is presented in the classical mount so by symmetry no $p-s$ conversion may occur. ${ }^{11}$ For the higher frequency mode, up to $95 \%$ of the incident power is absorbed by the wax, with the resonance half width being $0.56 \mathrm{GHz}$, while the lower frequency mode is less well coupled. This difference in depth may be partially attributed to the probability of coupling to the respective modes. A Fourier series of the form

$$
\begin{aligned}
A(x)= & a_{1} \sin \left(k_{g} x+\Phi_{1}\right)+a_{2} \sin \left(2 k_{g} x+\Phi_{2}\right) \\
& +\ldots \ldots \ldots+a_{N} \sin \left(N k_{g} x+\Phi_{N}\right)
\end{aligned}
$$

may be used to describe the grating where the amplitude coefficients $\left(a_{N}\right)$ determine the probability of each of the $N k_{g}$ diffraction events, and hence, control the coupling strength to each of the modes. However, for a dual pitch square wave profile with a repeat period of three grooves, the dominant terms are multiples of the $N=3$ term. ${ }^{4,5}$ Thus the $3 \boldsymbol{k}_{g}$ scattering process has a Fourier amplitude coefficient which is much greater than that of the $\boldsymbol{k}_{g}$ component introduced by the long period shallower grating. Hence, the probability of coupling to the higher frequency mode associated with a single $3 \boldsymbol{k}_{g}$ scattering event is greater than the probability of coupling to the lower frequency mode, which requires a multiscattering event.

Figure 3 shows the dispersion of the modes in the classical mount. The frequency of each resonant mode was obtained for six values of $\theta$ in the range $12^{\circ}<\theta<78^{\circ}$ and plotted against in-plane momentum $\boldsymbol{k}_{x}$. The higher frequency mode is remarkably non-dispersive, while the frequency of the lower mode reduces as $\boldsymbol{k}_{x}$ increases. Evaluation of the field profiles on resonance demonstrates the 21 $\mathrm{GHz}$ mode to be a strongly localized mode associated with standing waves in the shallower grooves of the grating profile, while the $19 \mathrm{GHz}$ mode is less localized in nature.

Figure 4 shows (a) $p$-polarized and (b) $s$-polarized experimental reflectivity data as a function of both frequency and azimuth at $\theta=52^{\circ}$. The nondispersive mode and dispersive lower frequency mode are clearly symmetric in character about $\phi=45^{\circ}$. It is apparent that unlike the monograting results ${ }^{5}$ which showed the modes supported by the dual period monograting to be non dispersive with $\theta$ only, the frequency and depth of the localized mode are now almost en-

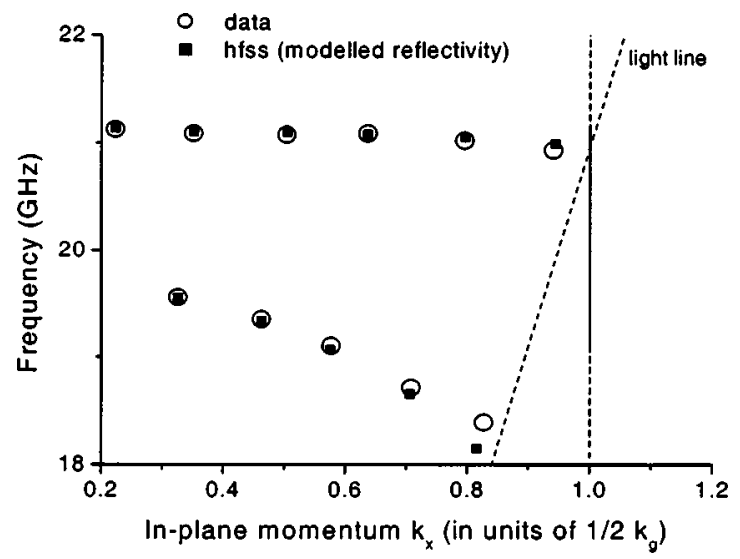

FIG. 3. The theoretical and experimental dispersion curves of the modes supported by the structure when presented in the classical mount. The vertical line represents the Brillouin zone boundary.

tirely independent of $\theta, \phi$, and polarization. This effect is due to the extra dimensionality that the bigrating possesses over the monograting. As $\phi$ increases, the projection of the incident wave vector in the direction of the grating wave vector decreases, and hence, the frequency of the lower mode increases to satisfy the momentum conservation criteria. The fourfold symmetry of the bigrating results in the frequency

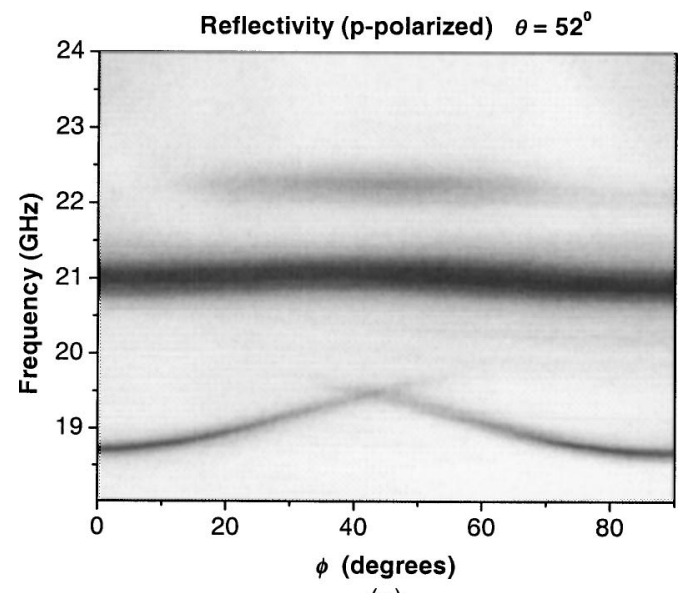

(a)

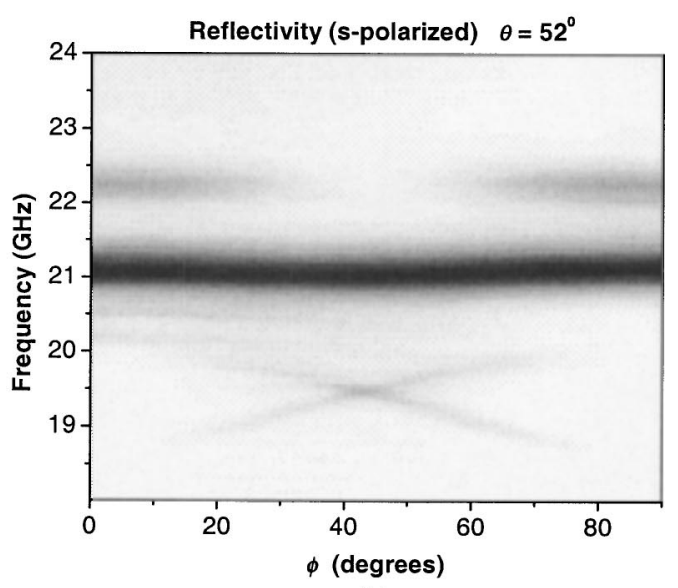

(b)

FIG. 4. (a) $p$-polarized and (b) $s$-polarized reflectivity data (experimental) as a function of both frequency and azimuth angle at a fixed polar angle of $52^{\circ}$. Light regions correspond to strong reflection while dark regions correspond to absorption.

Downloaded 17 Apr 2008 to 144.173.6.75. Redistribution subject to AIP license or copyright; see http://apl.aip.org/apl/copyright.jsp 


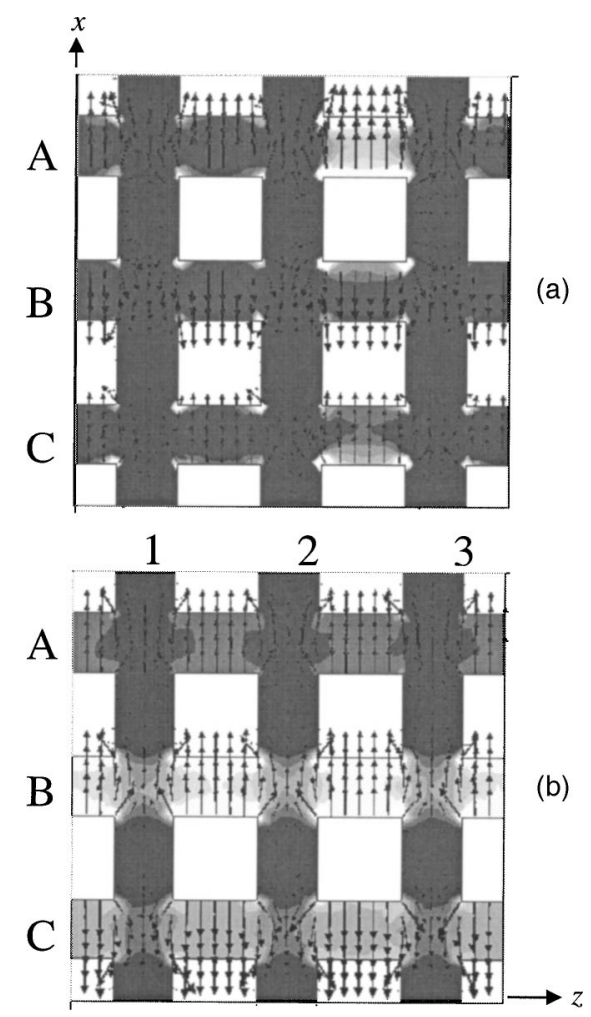

FIG. 5. The modeled electric vector (arrows) of the scattered field at the resonant frequency of (a) the $21 \mathrm{GHz}$ mode and (b) the $19 \mathrm{GHz}$ mode. Also shown are the relative field enhancements which range from dark gray $=8$ to white $=15$, averaged over $360^{\circ}$ of phase.

of the lower mode becoming degenerate at the symmetry angle $\phi=45^{\circ}$. Beyond $45^{\circ}$, coupling to the mode associated with the second orthogonal grating strengthens and further rotation of the sample results in a reduction of $\phi$ as measured with respect to the second grating wave vector, and a consequent reduction in frequency. Coupling at $\phi=90^{\circ}$ is therefore entirely associated with the second orthogonal corrugation.

From comparison of Figs. 4(a) and 4(b), the nondispersive but less well coupled $22.25 \mathrm{GHz}$ mode has its strongest coupling at $45^{\circ}$ for $p$-polarized incident radiation and at $0^{\circ}$ for the $s$-polarized case. This mode is associated with $(1,1)$ scattering. Coupling to the lower more dispersive mode is a maximum for $p$-polarized radiation at $0^{\circ}$ and reduces as $\phi$ is increased up to $45^{\circ}$. For $s$-polarized radiation the maximum coupling strength to the lower mode is centred at $\phi=45^{\circ}$. However, coupling to the nondispersive deep mode is possible for both $p$ and $s$ polarizations at all $\phi$. To help understand the different coupling characteristics of the two $(1,0)$ modes, the nature of the electromagnetic fields at each of the resonant frequencies are explored.

Figures 5(a) and 5(b) show the electric vector of the electromagnetic field (arrows) at the resonant frequency of the 21 and $19 \mathrm{GHz}$ modes, respectively, evaluated over a plane that cuts through the grating cavities at $1 / 3$ the depth of the deep grooves. Also shown are the relative field magnitudes. Grooves $I$ and $A$ are the slightly shallower grooves. Radiation is incident in the $x-y$ plane at an angle of $52^{\circ}$ to the normal and is $p$ polarized. The regions of high field occur within the cavities running parallel to the $z$ direction. Evaluation of the electric vector for the $21 \mathrm{GHz}$ mode over $360^{\circ}$ of phase shows the oscillating nature of the fields pertaining to grooves $A$ and $B$ to be antisymmetric while the field in groove $C$ leads that of $B$ by $90^{\circ}$. The field magnitude shows that the high field is confined to a region of the shallow groove $(A)$ bounded by two deep grooves. As a result, coupling to the nondispersive mode is dependent predominantly on field oscillation in the shallow grooves only. Thus, this nondispersive mode may be excited by either $p$ - or $s$-polarized radiation for values of $\theta$ up to and including normal incidence.

The fields of the more dispersive $19 \mathrm{GHz}$ mode are seen from Fig. 5(b) to be associated with the two deeper grooves $(B, C)$. Field magnitudes in these two regions are comparable, and the charge density oscillation is close to being in antiphase. For the $p$-polarized case at finite $\theta$ the incident plane wave is able to excite an antiphase oscillation at $\phi$ $=0^{\circ}$ due to the phase reversal of the incident electric vector providing a component of electric field in the $+x$ and $-x$ directions in the vicinity of adjacent grooves simultaneously. However, in the $s$-polarized case the phase reversal of the incident electric vector occurs in the $z$ direction, perpendicular to the direction of mode propagation and thus at $\phi=0^{\circ}$ it may not excite this mode.

In summary a reflectivity study of a dual pitch nondiffracting metal bigrating in the microwave regime is presented. The dual periodicity and fourfold symmetry of the bigrating results in a structure which not only supports a remarkably nondispersive SP mode that is largely independent of $\theta$, but unlike previous dual period gratings is independent of both azimuth angle and polarization of the incident beam. This mode is shown to be an extremely efficient absorber of incident radiation at frequencies which may be tuned by the physical parameters of the grating, clearly demonstrating the potential of selectively absorbing designer surfaces. Coupling to a further flatbanded mode associated with $(1,1)$ scattering is also noted. Experimental data show excellent agreement with model predictions.

The authors are grateful for support from the Engineering and Physical Sciences Research Council and the provision of a CASE award by QinetiQ (Farnborough) for M.J.L. This work was carried out as part of Technology Group 09 of the MoD Corporate Research Fund.

${ }^{1}$ R. W. Wood, Philos. Mag. 4, 396 (1902).

${ }^{2}$ H. Raether, Surface Plasmons (Springer, Berlin, 1988).

${ }^{3}$ R. Petit, Electromagnetic Theory of Gratings (Springer, New York, 1980).

${ }^{4}$ W.-C. Tan, J. R. Sambles, and T. W. Priest, Phys. Rev. B 61, 13177 (2000).

${ }^{5}$ A. P. Hibbins, J. R. Sambles, and C. R. Lawrence, Appl. Phys. Lett. 80, 2410 (2002)

${ }^{6}$ G. H. Derrick, R. C. Mc Phedran, D. Maystre, and M. Neviere, Appl. Phys. 18, 39 (1979).

${ }^{7}$ N. Kawatsuki and M. Uetsuki, Jpn. J. Appl. Phys., Part 1 29, 2420 (1990).

${ }^{8}$ A. P. Hibbins, J. R. Sambles, and C. R. Lawrence, Phys. Rev. E 61, 5900 (2000).

${ }^{9}$ A. P. Hibbins, J. R. Sambles, and C. R. Lawrence, J. Appl. Phys. 86, 1791 (1999).

${ }^{10}$ www.ansoft.com/products/hf/hfss/index.cfm.

${ }^{11}$ S. J. Elston, G. P. Bryan-Brown, T. W. Preist, and J. R. Sambles, Phys. Rev. B 44, 3483 (1996). 